\title{
Systematic analysis of hsa-miR-363 gene overexpression pattern in endometrial stromal cells
}

\author{
WENQU LI ${ }^{1 *}$, XUEMEI FAN ${ }^{1 *}$, MI ZHANG ${ }^{1 *}$, LEI HUANG ${ }^{2}$, SHANSHAN LV $^{3}$, LUYU WANG $^{4}$, \\ YING WU ${ }^{1}$, CHENCHENG DAI $^{1}$, JUAN XU $^{1}$, PENGFEI XU ${ }^{1}$, ZIYI FU ${ }^{1}$, \\ XUEMEI JIA $^{1}$ and XIAOYAN SHI ${ }^{1}$

\begin{abstract}
${ }^{1}$ Department of Gynecology, Affiliated Obstetrics and Gynecology Hospital of Nanjing Medical University, Nanjing Maternal and Child Health Care Hospital, Nanjing, Jiangsu 210004; ${ }^{2}$ Department of General Surgery, The Affiliated Drum Tower Hospital of Nanjing University Medical School, Nanjing, Jiangsu 210008; ${ }^{3}$ Nanjing University of Chinese Medicine, Nanjing, Jiangsu 210029; ${ }^{4}$ Soochow University, Soochow, Jiangsu 215004, P.R. China
\end{abstract}

Received March 9, 2016; Accepted January 18, 2018

DOI: $10.3892 /$ ijmm.2018.3840

\begin{abstract}
Endometriosis is a benign disease, but has invasion and metastasis characteristics similar to malignant tumors. Clinically, it is a difficult problem of gynecological clinical treatment for its high recurrence rate. It has been confirmed that miR-363 was downregulated in endometriosis tissues and miR-363 overexpression inhibited the invasion ability of endometrial stromal cells (ESCs). In order to explore the potential mechanism of miR-363-reduced ESC migration and invasion progression, we sought to demonstrate the targeted mRNA expression levels of miR-363 through microarray, and performed cluster analysis to identify potential functions of these targeted genes in ESCs. The wound migration assay showed that there was an observable trend of cell migration potential decrease after transfection with hsa-miR-363. The qRT-PCR result showed that compared to miR-363 negative control cell group, miR-363 was upregulated 3,264.58-fold after miR-363 lentiviral transfection in miR-363 mimics group. The microarray data showed that compared to ESCs miR-363 negative control cell group, 249 genes were upregulated in ESCs miR-363 mimics cells group, and 139 genes were downregulated. Gene Ontology analysis and the pathway analysis data demonstrated that these target genes are mainly involved in cell migration, cell adhesion and invasion, proliferation, apoptosis, alteration of endometrial cells and some related signaling pathways. Our study explored the gene expression pattern after miR-363 overexpression,
\end{abstract}

Correspondence to: Dr Xuemei Jia or Dr Xiaoyan Shi, Department of Gynecology, Affiliated Obstetrics and Gynecology Hospital of Nanjing Medical University, Nanjing Maternal and Child Health Care Hospital, 123 Tianfeixiang street, Mochou Road, Nanjing, Jiangsu 210004, P.R. China

E-mail:xmjia@njmu.edu.cn

E-mail: shixy019@vip.sina.com

*Contributed eqully

Key words: gene microarray, endometriosis, endometrial stromal cell, miR-363 which could expand the insights into the miR-363 function and molecular mechanisms in endometriosis.

\section{Introduction}

Endometriosis is a benign gynecological disease, which is characterized by the presence of functional endometrial glands and stroma outside the uterine cavity. The characteristics of endometriosis, for example invasion and metastasis ability, are similar to the malignant tumors, and endometriosis affects up to $20 \%$ of women of reproductive age who suffer from dysmenorrheal, chronic pelvic pain, subfertility and dyspareunia (1). It decreases the life quality of the affected women, and the clinical prognosis of endometriosis is still poor due to the high recurrence rate. Present medications only ameliorate symptoms and cause significant side effects such as osteoporosis at the same time. Therefore, novel therapeutic agents that suppress endometriotic lesion establishment and growth to reduce the high burden conferred by this disease are required (2). The pathogenesis of endometriosis is not fully characterized. Sampson's theory of retrograde menstruation, first described in 1927, remains the most accepted theory for the origin of this disease (3). According to Sampson's theory, endometrial stromal cells (ESCs) could escape from the uterine cavity and adhere to the peritoneal surface to mediate the invasion and metastasis to the target organs (4).

In the last decade, we found that various cellular physiological and pathological processes (including cell cycle, metabolism, cell-cell communication, cell survival and apoptosis, immune responses and oncogenesis) were induced or promoted due to dozens of genes targeted by a single miRNA through the perfect or partial base pairing with the 3'-untranslated region (3'-UTR) of the target mRNAs $(5,6)$. Additionally, studies suggest that miRNAs play an important role in endometriosis (7-9). For example, miR-210 can promote the proliferation, resistance to apoptosis and VEGF production of endometriosis through STAT3 activation (6). It can be seen that miRNAs have important roles in pathological processes of endometriosis, though the mechanisms responsible for endometriosis remain largely unknown. 
miRNAs belong to a large class of small, single-stranded, non-coding RNAs, molecules approximately 18 to 25 nucleotides in length. They can bind to mRNA, preventing translation and accelerating mRNA deadenylation and subsequent degradation, thus having a gene silencing effect (10). In a previous study (ref. ?), miR-363 was found significantly downregulated in endometriosis tissues than in normal tissues by microRNA chip technology. We supposed that miR-363 plays a significant role in endometriosis mediating target genes. However, the mechanism of miR-363 in endometriosis process remains largely unexplored.

In this study, we sought to determine the expression pattern of miR-363 targeted mRNAs by sequence-specific base pairing on the 3'-UTR, and explored their potential functions and pathways, which are involved in the biological behavior of human endometriosis. This study may afford new clues for understanding the pathogenesis of endometriosis, and miR-363 may serve as a therapeutic option in endometriosis treatment.

\section{Materials and methods}

Cell lines and cell culture. The human ESC line was obtained from the American Type Culture Collection (ATCC; Manassas, VA, USA). Cells were cultured in Dulbecco's modified Eagle's medium (DMEM) supplemented with $10 \%$ (v/v) fetal bovine serum (FBS), $100 \mathrm{U} / \mathrm{ml}$ penicillin and $100 \mathrm{U} / \mathrm{ml}$ streptomycin (all from Gibco Life Technologies, Carlsbad, CA, USA) at $37^{\circ} \mathrm{C}$ in a humidified atmosphere containing $5 \% \mathrm{CO}_{2}$.

Lentiviral transfection of ESC cells. ESC cells were seeded into 6-well plates and incubated overnight, reached $60-80 \%$ confluence before transfection. Transient transfection of ESC with mimics for hsa-miR-363 or counterpart negative control used lentiviral vector (LV-3-GFP-PURO; Shanghai GenePharma Co., Ltd., Shanghai, China) according to the manufacturer's instructions. The experiments were classified into two groups, hsa-miR-363 (or miR-363 mimics) group, and negative control (NC) group. After $24 \mathrm{~h}$ post-transfection, the medium was removed and the fresh culture medium with $10 \%$ FBS was injected. The transfection efficiency of lentiviral vector was observed by fluorescence microscope at 48 and $72 \mathrm{~h}$ after transfection.

Wound migration assay. A density of $1 \times 10^{5}$ ESC cells/ well were seeded into 6 -well plates. When they were approximately $80 \%$ confluent, ESC cells were transfected with hsa-miR-363 or negative control vector for $24 \mathrm{~h}$. The monolayer was scratched with a sterile $10 \mu \mathrm{l}$ pipette tip, and rinsed with phosphate-buffered saline (PBS) to remove cellular debris. Subsequently, fresh medium was added and cells were incubated for $48 \mathrm{~h}$ to allow time for migration into the cell-free area. The images of the migratory cells were captured at times 0,24 and $48 \mathrm{~h}$ post-wounding using an Olympus IX70 microscope equipped with digital camera (Olympus America Inc., Melville, NY, USA). Images were analyzed by the TScratch software (CSE, Switzerland). Experiments were run in triple independent repeats.

Gene microarray analysis. Standard gene microarray was performed to analyze the gene expression characteristics after miRNA-363 overexpression (11). After ESC cells were transfected with hsa-miR-363 mimics or counterpart negative control lentivirus vector for $72 \mathrm{~h}$, respectively, total RNA was isolated using TRIzol reagent (Invitrogen Life Technologies, Carlsbad, CA, USA) according to the manufacturer's instructions. Total RNA was extracted using Qiagen RNeasy Mini kit and RNase-free DNase I (both from Qiagen GmbH, Hilden, Germany) according to the manufacturer's instructions. The purity and concentration of total RNA were measured by the UV absorbance at 260 and $280 \mathrm{~nm}(260 / 280 \mathrm{~nm})$. Global gene expression was detected using Affymetrix GeneChip Human Gene 1.0 ST array (Affymetrix, Inc., Santa Clara, CA, USA). About 500 ng of total RNA were employed in each experiment. Briefly, total RNA of each sample was amplified using the Ambion WT Expression kit (Applied Biosystems, Foster City, CA, USA) according to the manufacturer's instructions. Approximately $5.5 \mu \mathrm{g}$ of generated cDNA was fragmented and labeled using GeneChip3 WT Terminal Labeling kit and Control kit (both from Affymetrix, Inc.). Labeled cDNA target was hybridized to the Affymetrix GeneChip Human Gene 1.0 ST array using GeneChip3 Hybridization, Wash, and Stain kit (Affymetrix, Inc.) at $45^{\circ} \mathrm{C}$ for $16 \mathrm{~h}$, then hybridized arrays were washed and stained on the Affymetrix GeneChip Command Console (AGCC; Affymetrix, Inc.), and scanned on a GeneChip2 Scanner 3000 7G (Affymetrix, Inc.). The acquired array images were analyzed by Affymetrix GeneChip Operating Software (GCOS). Affymetrix expression console software was used to perform quantile normalization and subsequent data processing. Data transformation was applied to set all negative raw values at 1.0 , followed by quantile normalization. A filter on low gene expression was used to keep only the probes expressed in at least one sample. Differentially regulated genes were identified via fold change filtering.

Real-time PCR quantification of miR-363 and mRNAs. Total RNA was isolated from cultured cells using TRIzol reagent (Invitrogen Life Technologies) according to the manufacturer's instructions. miR-363 was reverse transcribed to cDNA by using TaqMan MicroRNA Reverse Transcription kit with specific probes according to the TaqMan MicroRNA assay protocol (both from Applied Biosystems), and human snRNA U6 as an endogenous control. qRT-PCR was performed using a ABI 7900 PCR system (Applied Biosystems). The $20 \mu \mathrm{l}$ reaction volume included $100 \mathrm{ng}$ RT product, $10 \mu \mathrm{l}$ 2X TaqMan Universal Master mix II and $1 \mu \mathrm{l}$ primers, and was performed for $2 \mathrm{~min}$ at $50^{\circ} \mathrm{C}, 10 \mathrm{~min}$ at $95^{\circ} \mathrm{C}, 15 \mathrm{sec}$ at $95^{\circ} \mathrm{C}$ and $1 \mathrm{~min}$ at $60^{\circ} \mathrm{C}$ for 40 cycles followed by the thermal denaturation protocol. All reactions were done in triplicate. The expression of miR-363 was defined based on the threshold cycle $(\mathrm{Ct})$, and relative expression levels were calculated as $2^{-\Delta \Delta \mathrm{Ct}}$ after normalization with reference to expression of snRNA U6.

For quantification of mRNA, the purified total RNA $(2 \mu \mathrm{g})$ was reverse transcribed using the PrimeScript RT Master mix (Perfect Real Time) (cat. no. RR036A; Takara Bio, Inc., Otsu, Japan), and qPCR was performed on an ABI 7900 PCR system using Power SYBR-Green PCR Master mix (2X) (both from Applied Biosystems). GAPDH was used as a normalization control. Primers for amplification are listed in Table I. Experiments were performed in triplicates in three 
Table I. Primers for amplification.

\begin{tabular}{lll}
\hline Target genes & \multicolumn{1}{c}{ Forward primers } & \multicolumn{1}{c}{ Reverse primers } \\
\hline ITGA5 & AGGACAGATGCCACACAAGG & CTGCCTGGATTAGGTGCCAA \\
ITGA6 & GTGGCTATTCTCGCTGGGAT & AGAGCGTTTAAAGAATCCACACT \\
FHL2 & GGGGAGACTGGTTGCTGAAA & AGGGTCTCAAAGCACACCAC \\
PRKCB & GGGTGTCCTGTCCAGAAAGT & CTTCCTGAGAGCATGTCCAA \\
SNORD15A & GATGTTCTCTTTGCCCAGGT & TTCAACCAGGGCTCTTTAAACT \\
GGT7 & CCACTCCTGCCTGTCTATGA & GCCACAATGAAGTCATCAGG \\
SERPINA9 & AGAACTTCCCATTCCTGGTG & ACGGCATCTCCCTTGTAATC \\
SERPINB6 & AGGAGAACACCGAGGAGAGA & TTCCTTGCCAACATATGGAA \\
ABHD14B & ATATGACCCTTCGCTTGAGG & GTGTGGAGGCTCATGTATGC \\
ADAM19 & CAAGGGCCAACACCTTATTT & AGGTCGCTTCTTGGTCTGTT \\
GAS7 & ATCTGGCTTTCTCAGCCACT & GCCGAATGAGGTAGTTCCAT \\
FAM198A & CAGATGGACCCAGTGTTCTG & TCGTCCCTGAAGAGTGTGAG \\
EGR2 & GAGTTGGGTCTCCAGGTTGT & CACCGGGTAGATGTTGTCAG \\
\hline
\end{tabular}

independent experiments. Errors are reported as mean \pm SD. The alteration in mRNA expression was calculated as the fold change relative to control. Student's t-tests were used for statistical analyses with two-tailed distributions and two-sample unequal variance parameters.

Gene Ontology (GO) and Kyoto Encyclopedia of Genes and Genomes (KEGG) analysis. GO function and KEGG enrichment for differentially expressed genes were used to identify the significantly enriched biological terms and pathways (11). The Database for Annotation, Visualization and Integrated Discovery (DAVID) (12) was applied to perform GO function and KEGG enrichment for differentially expressed genes. Putative genes that were downregulated more than 2-fold with a P-value $<0.05$ after miR-363 overexpression compared to miR-363 NC group were considered as differentially expressed and selected to submit to DAVID Bioinformatics Resources 6.7, NIAID/NIH (http://david.abcc.ncifcrf.gov/). The overall functions regulated by these miR-363 modulated genes were identified by functional annotation clustering and ranked by enrichment scores.

Statistical analysis. Each experiment was done at least in triplicate. All statistical analyses were carried out by two-tailed Student's t-test. Numerical data were presented as means \pm standard deviation (SD). A P-value $<0.05$ was considered statistically significant. Computer-based calculations were conducted using SPSS version 20 (SPSS, Inc., Chicago, IL, USA).

\section{Results}

The efficiency of lentiviral transfection in ESC. To determine the efficiency of lentiviral transfection and the miR-363 expression level in ESC, fluorescence microscope and qRT-PCR were used at $96 \mathrm{~h}$ after transfection. The results showed that over $90 \%$ of cells expressed green fluorescent protein (GFP) (Fig. 1A). Compared to ESC miR-363 NC cell group, the expression levels of miR-363 in ESC miR-363 mimics cell group were significantly upregulated 3,264.58-fold (Fig. 1B), and in a lentivirus dose-dependent manner. These results confirmed the transfection efficiency of lentiviral vector with miR-363 in ESC.

Wound migration assay. ESC cells were transfected with hsa-miR-363 or NC vector for $24 \mathrm{~h}$. Subsequently, scratch wound migration assay was performed. There was an observable trend of decrease of the cell migration potential at $24 \mathrm{~h}$ after transfection (Fig. 2).

Differentially expressed genes. In order to explore the potential mechanism of miR-363 in human breast cancer, gene microarray was carried out to detect the whole coding gene expression pattern after miR-363 overexpression. The gene expression profiles showed that, compared to ESC miR-363 NC cell group, 249 genes were upregulated in ESC miR-363 mimics group, and 139 genes were downregulated (fold change threshold was $\geq 2.0$ ) (Fig. 3A). Although we used a 2-fold change as our threshold in gene expression, only some of these genes exhibited $>2.0$-fold change in gene expression and these differences reached statistical significance $(\mathrm{P}<0.05)$ (Fig. 3B). To assess the validity of the microarray data, real-time quantitative PCR was performed to compare gene expression levels between the two cell groups. We selected 10 genes from array dataset (raw intensity of ESC miR-363 NC was $\geq 100$ ) and 3 genes which were not downregulated $>2.0$-fold in the dataset, but had great connections to the endometriosis through bioinformatics analysis of miR-363 to validate the microarray data, all of which were downregulated in ESC miR-363 mimics cell group. RT-PCR data was consistent with the results of the microarray, suggesting that the dataset obtained from the microarray analysis accurately reflects gene expression differences between ESC miR-363 NC cells and ESC miR-363 mimics cells (Fig. 3C).

GO and pathways mapping analysis. We performed GO and KEGG analysis to identify the biological pathways of these differentially expressed genes in the dataset. GO analysis is a functional analysis associating differentially expressed 

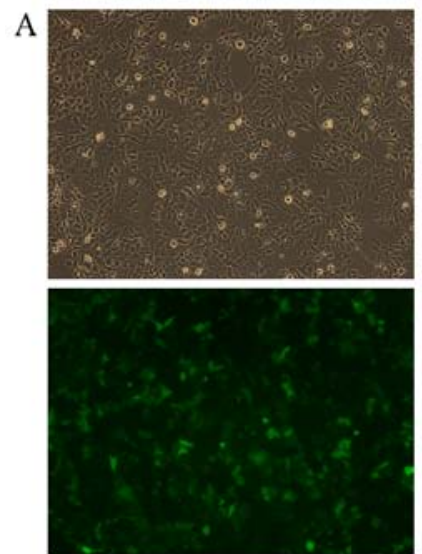

miR-363 NC
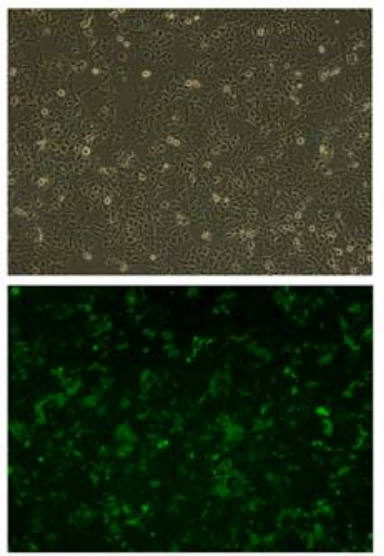

miR-363 mimics

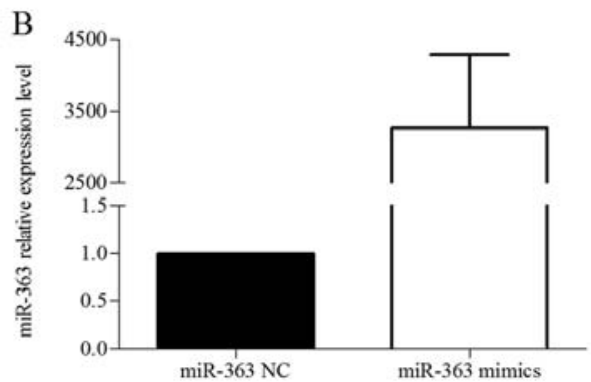

Figure 1. (A) The efficiency of lentiviral transfection was determined by fluorescence microscope at $96 \mathrm{~h}$ after transfection, and over $90 \%$ of cells expressed green fluorescent protein (GFP). (B) Compared to endometrial stromal cells (ESC) NC cell group, the expression levels of miR-363 in ESC miR-363 mimics cell group were significantly upregulated 3,264.58-fold by qRT-PCR.
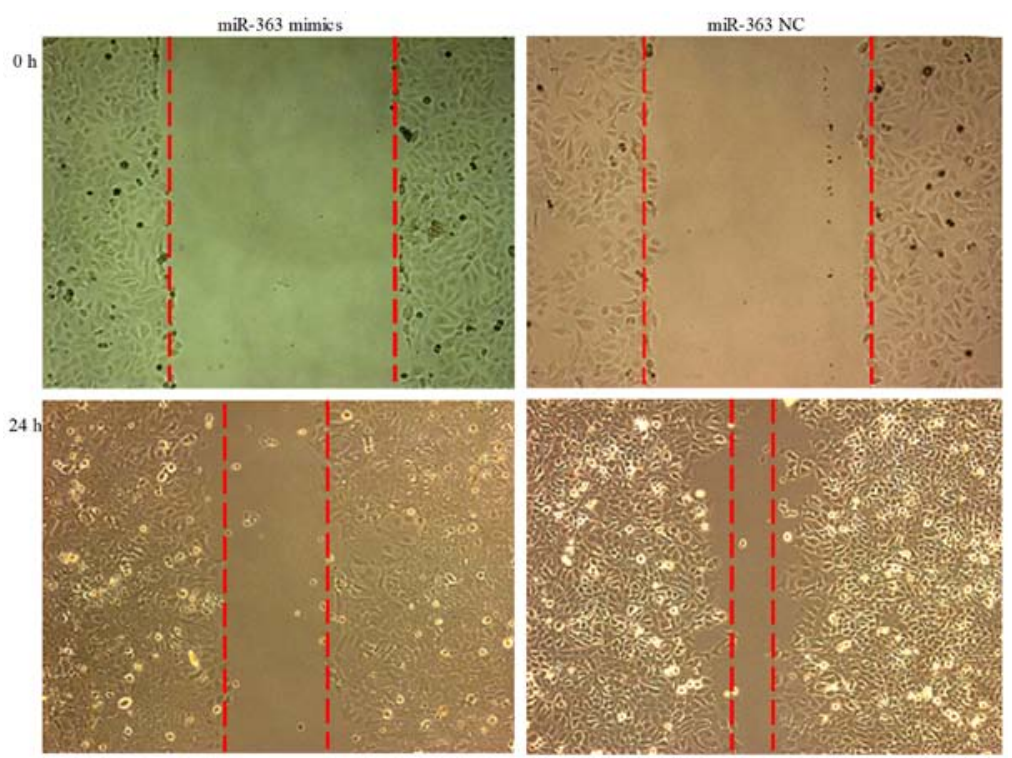

Figure 2. Compared to NC group, there was an observable trend of decrease of cell migration potential at $24 \mathrm{~h}$ after transfection of miR-363 mimics.

genes with GO categories. We employed a GO approach using DAVID tools as an unbiased method for identifying the predominant biological process and pathways representing the target genes. GO analysis result showed that the genes were enriched in the following biological processes: regulation of cell death, mitotic cell cycle, regulation of apoptosis, cell cycle, mitosis, negative regulation of gene expression, regulation of cell development, regulation of cell motion, regulation of growth, and regulation of translation (Fig. 4A); the gene production mainly composed of cytoskeleton, mitochondrion, nucleolus, centrosome, spindle, nuclear body, transcriptional repressor complex, actin cytoskeleton, transcription factor complex and adherens junction (Fig. 4B); the molecular functions of these genes included nucleotide binding, ATP binding, ribonucleotide binding, DNA binding, nucleosidetriphosphatase regulator activity, cytoskeletal protein binding, transcription factor binding, transcription activator activity, actin binding, and transcription regulator activity (Fig. 4C).
Then, we used the KEGG pathway mapping tools to identify the biological pathways of these genes, included p53 signaling pathway, pathways in cancer, MAPK signaling pathway, regulation of actin cytoskeleton, focal adhesion, adherens junction, RNA polymerase, mTOR signaling pathway, apoptosis, amino sugar and nucleotide sugar metabolism, Wnt signaling pathway, Notch signaling pathway and GnRH signaling pathway (Fig. 4D).

\section{Discussion}

The invasion and metastasis characteristics play important roles in endometriosis. Several mechanisms have been proved to connect to the invasion and metastasis characteristics and the alterations to miRNA expression levels is a new point in endometriosis. In our study, the endometriosis cell line ESC was widely used as an in vitro model for study of endometriosis. The invasion and metastasis ability of ESC was mainly 


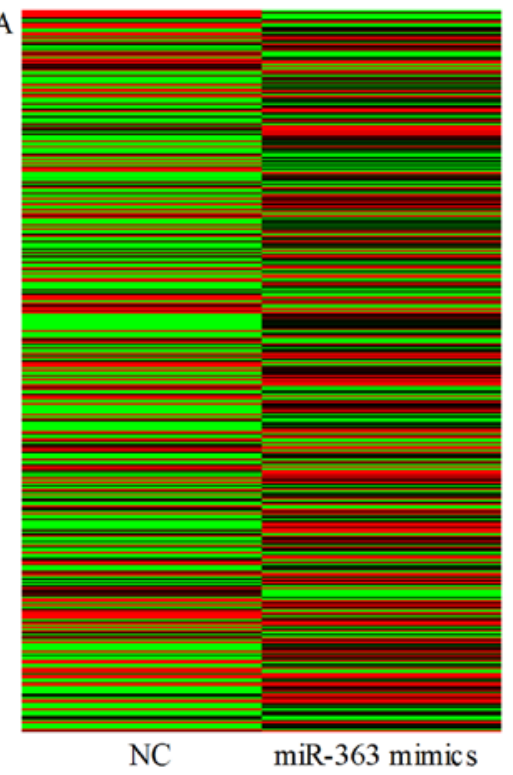

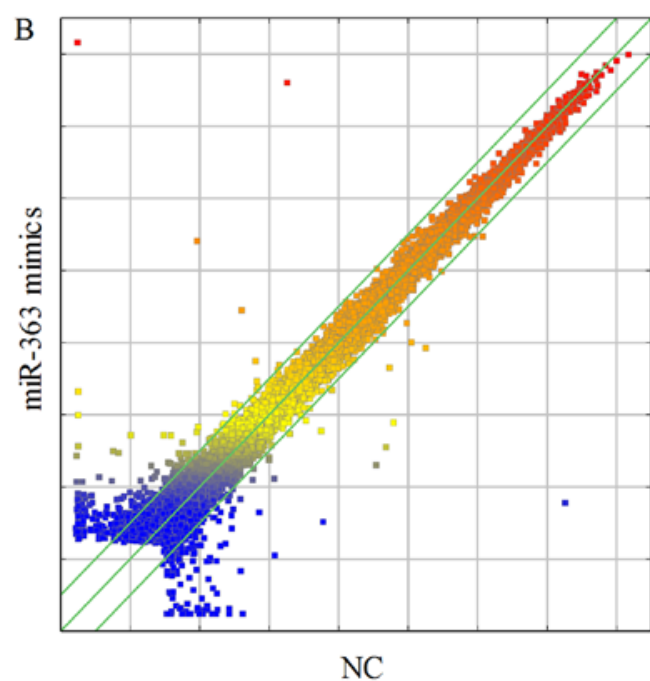

$\mathrm{NC}$

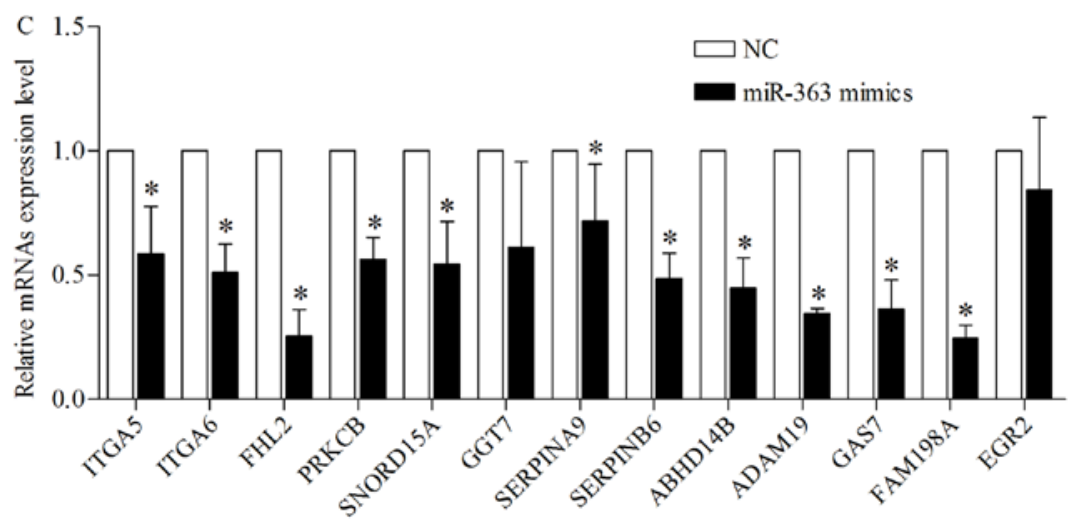

Figure 3. Differentially expressed genes in endometrial stromal cells (ESC) miR-363 mimics cells and ESC NC cells. (A) Heat map shows regulated genes by miR-363 that significantly increased or decreased after lentiviral transfection. Dot plot overview of up and downregulated genes by miR-363 after lentiviral transfection are compared to miR-363 NC group control. (B) Green lines represent unchanged (middle), 2-fold upregulated (upper-left) or 2-fold downregulated (lower right) genes. To confirm the validity of the microarray data, qRT-PCR was used to compared gene expression levels between the two cell groups. Compared to ESC NC cells, 13 genes (ITGA5, ITGA6, FHL2, PRKCB, SNORD15A, GGT7, SERPINA9, SERPINB6, ABHD14B, ADAM19, GAS7, FAM198A and EGR2) were significantly downregulated in ESC miR-363 mimics cell group (calculated as $2^{-\Delta \Delta C t}$ ). (C) The results of qRT-PCR were consistent with the results of the microarray. ${ }^{\mathrm{P}}<0.05$, compared to NC.

adjusted by its own and local microenvironment, including multiple hormones, cytokines, growth factors, extracellular matrix glycoprotein and various kinds of transcription factors, and involving a large amount of signaling pathways, such as MAPK, PI3K/Akt signaling pathways (13-16).

miR-363 has been found to participate in regulating cancer biological progress. Several studies have shown that miR-363 can regulate the invasion and metastasis of tumors $(17,18)$. Whereas, there is no study exploring the function of miR-363 in endometriosis. In our GO and KEGG analysis, we found that the target genes of miR-363 participated in many significant biological processes, cellular components, molecular functions, and signaling pathways. All of the above were involved in migration, cell adhesion and invasion, proliferation, apoptosis, alteration of endometrial cells.

The target gene ITGA5 is a member of the cell adhesion molecule family that acts on both cell-cell and cell-substratum adhesion. They can promote cell attachment to proteins within ECM and enhance cell migrationand invasion (1). Vernet-Tomás et al found integrins could be mediated endometrial cells and peritoneal mesothelial cell adhesion, causing pelvic adhesion extensively (19). According to Giannelli et al, this family can also activate the ERK and JNK signaling pathway in MAPK signaling pathway, promote the adhesion and motion of endometrial cells (20).

In the last few years, aberrant activation of MAPK was found to play important roles in pathological processes of endometriosis progression (21). Such as, aberrant activation of ERK was shown to be involved in interleukin (IL)-1 $\beta$ and macrophage migration inhibitory factor-induced cyclooxygenase- 2 expression, prostaglandin $\mathrm{E}_{2}\left(\mathrm{PGE}_{2}\right)$-induced fibroblast growth factor 9 (FGF9) expression (21-25). In addition, activation of Akt independently can regulate cell invasion, metastasis, angiogenesis, and survival $(26,27)$. Recently, associated studies reported PI3K/AKT signaling 

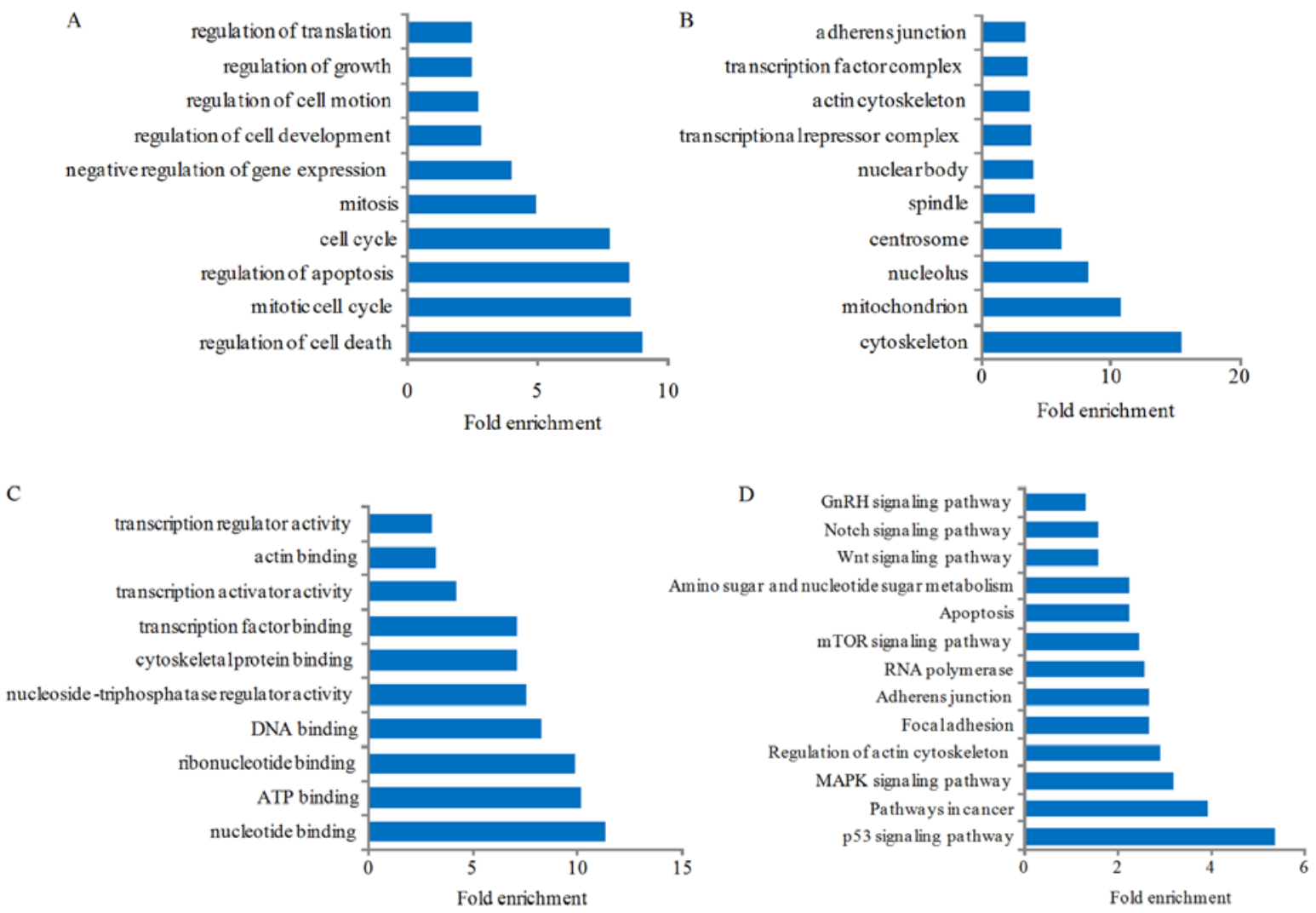

Figure 4. Gene Ontology (GO) and Kyoto Encyclopedia of Genes and Genomes (KEGG) analysis of the differentially expressed genes after miR-363 overexpression. GO analysis result show that these genes were enriched in the following biological processes: (A) regulation of cell death, mitotic cell cycle, regulation of apoptosis, cell cycle, mitosis, negative regulation of gene expression, regulation of cell development, regulation of cell motion, regulation of growth, and regulation of translation; (B) these genes' production mainly composed into cytoskeleton, mitochondrion, nucleolus, centrosome, spindle, nuclear body, transcriptional repressor complex, actin cytoskeleton, transcription factor complex and adherens junction; (C) the molecular functions of these genes included nucleotide binding, ATP binding, ribonucleotide binding, DNA binding, nucleoside-triphosphatase regulator activity, cytoskeletal protein binding, transcription factor binding, transcription activator activity, actin binding, and transcription regulator activity; (D) the biological pathways of these genes, included p53 signaling pathway, pathways in cancer, MAPK signaling pathway, regulation of actin cytoskeleton, focal adhesion, adherens junction, RNA polymerase, mTOR signaling pathway, apoptosis, amino sugar and nucleotide sugar metabolism, Wnt signaling pathway, Notch signaling pathway, and GnRH signaling pathway.

pathway was activated in endometriosis. Especially, p-AKT was highly expressed in endometriotic stromal cells, leading to the reduced decidualization and cell survival (26,28-30).

The mechanism of regulating cellular proliferation in endometriosis is considered to be generally the same as normal endometrium, as a result of the interaction between gender steroids and their corresponding receptors. As an immunohistochemical marker of cell proliferation, the expression of proliferating cell nuclear antigen in endometriotic lesions is much higher than eutopic and normal endometrium, indicating a higher proliferative activity. In addition, the cyclical changes in cellular proliferation observed in eutopic and normal endometrium are not discovered in endometriotic lesions (1).

Apoptosis is a complex process, regulated by several multifunctional mediators. As a programmed cell death it kills unwanted cells without inducing an immune response or inflammation due to these multifunctional mediators. In normal human endometrium, apoptosis has been reported to appear in the mid secretory phase, increasing in the late secretory phase, and is maximal during the menstrual phase. Because of programmed cell death, endometrial cells from healthy women during menstruation do not survive in ectopic locations (1,31-33).
It has been demonstrated that apoptotic events seemed to be decreased in endometriotic lesions compared with corresponding eutopic and normal endometrium, on the basis of endometriosis. Impaired sensitivity of the endometrial tissues to spontaneous apoptosis leads to abnormal implantation and growth of endometrial tissues in ectopic places (1). It has been suggested that the concordant endometrial expression of many genes that trigger the activation of apoptotic and cell-cycle signaling were identified. The activation of antiapoptotic genes and the inhibition of proapoptotic ones may contribute to the resistance of endometriotic cells to apoptosis (1,31-34).

Many miRNAs have been predicted to regulate the expression of genes with functions in apoptosis and cell cycle regulation of several cancer cells. However, limited evidence exists to support the regulatory functions of miRNAs in proliferative response of normal, differentiated quiescent cells (31,35-37). Several studies have suggested that remodeling of the actin cytoskeleton is required for the cell adhesion and movement. Via the restructuring of the cytoskeleton, the cyclic architectural modifications observed in the endometrium are achieved. It is confirmed that cytoskeletal protein is related to the endometriotic cell proliferation, adhesion, invasion and apoptosis (1). 
$\mathrm{Xu}$ et al found that cytoskeletal protein confilin 1 (CFL1) was overexpressed in endometriotic lesions and eutopic endometrium with endometriosis. Silencing the CFL1 gene in endometriotic cells can attenuate their proliferation, adhesion and invasion. On the contrary, once the CFLl gene is upregulated, the cell proliferation, adhesion and invasion increased $(1,38)$. As endometriosis is considered to be a multigenic disease with complex genetic traits, differences in hormonal regulation of the endometria is inferred as a result of an abnormal gene expression pattern and may be responsible for adhesion and invasion properties of the shed endometrial fragments (1). Absenger et al confirmed a highly regulated cysteine-rich 61 (Cyr61) gene in endometrium from women with endometriosis, from the results of microarray technology (39).

MicroRNAs are small non-coding RNAs of 18-25 nucleotides, which post-transcriptionally regulate gene expression and can control a broad spectrum of normal and pathological cellular functions via binding to their target mRNAs. Numerous diseases, including benign and malignant gynecological disorders, are associated with abnormal miRNA expression. Recently, researchers identified that miRNAs relevant to molecular pathways were associated with pathogenesis of endometriosis via conducting the miRNA expression profiles of endometrium from women with endometriosis $(9,8,40)$.

In conclusion, we found that miR-363 was associated with migration, cell adhesion and invasion, proliferation, apoptosis, alteration of endometrial cells in endometriosis and there were 139 potential target genes of miR-363 via microarray analysis. Through GO and KEGG pathways analysis, we also identified the biological processes and signaling pathways involved in endometriosis of ESC miR-363 mimics cell line. These results provided us a systematic understanding and global view of the function of overexpression miR-363 related to the process of endometriosis. Future investigation should be targeted to the biological processes and signaling pathways that have been identified. miR-363 may be a novel biomarker for the endometriosis prediction and prognosis, while more efforts should be done to identify the mechanism of miR-363 regulation of endometriosis.

\section{Acknowledgements}

Not applicable.

\section{Funding}

This study was financially supported by the National Natural Science Foundation of China (nos. 81401182, 81202007 and 81302304) and the Science and Technology Development Foundation of Nanjing Medical University (no. 2012NJMU185).

\section{Availability of data and material}

The datasets used and/or analyzed during the current study are available from the corresponding author on reasonable request.

\section{Authors' contributions}

WL performed gene microarray analysis, and was a major contributor in writing the manuscript. XF performed the ESC cell culture and lentiviral transfection. MZ performed the wound migration assay and real-time PCR. LH participated in gene microarray analysis and the manuscript modification. SL participated in gene microarray analysis and the manuscript modification. LW performed the related literature collection. YW analyzed the experiment data. CD participated in the ESC cell culture and lentiviral transfection. JX participated in the manuscript modification and the experiment data analysis. PX and ZF performed gene ontology and pathways mapping analysis. XJ performed the management of the experiment and foundation. XS performed personnel assignment and the manuscript modification. All authors read and approved the final manuscript.

\section{Ethics approval and consent to participate}

Not applicable.

\section{Patient consent for publication}

Not applicable.

\section{Competing interests}

Authors declare they have no competing interest.

\section{References}

1. Jiang QY and Wu RJ: Growth mechanisms of endometriotic cells in implanted places: a review. Gynecol Endocrinol 28: $562-567,2012$

2. Gao X, Outley J, Botteman M, Spalding J, Simon JA and Pashos CL: Economic burden of endometriosis. Fertil Steril 86: 1561-1572, 2006

3. Sampson J: Peritoneal endometriosis due to menstrual dissemination of endometrial tissue into the peritoneal cavity. Am J Obstet Gynecol 14: 422-469, 1927.

4. Nair AS, Nair HB, Lucidi RS, Kirchner AJ, Schenken RS, Tekmal RR and Witz CA: Modeling the early endometriotic lesion: mesothelium-endometrial cell co-culture increases endometrial invasion and alters mesothelial and endometrial gene transcription. Fertil Steril 90 (Suppl 4): 1487-1495, 2008.

5. Bartel DP: MicroRNAs: genomics, biogenesis, mechanism, and function. Cell 116: 281-297, 2004.

6. Okamoto M, Nasu K, Abe W, Aoyagi Y, Kawano Y, Kai K, Moriyama M and Narahara H: Enhanced miR-210 expression promotes the pathogenesis of endometriosis through activation of signal transducer and activator of transcription 3. Hum Reprod 30: 632-641, 2015.

7. Teague EM, Print CG and Hull ML: The role of microRNAs in endometriosis and associated reproductive conditions. Hum Reprod Update 16: 142-165, 2010.

8. Ohlsson Teague EM, Van der Hoek KH, Van der Hoek MB, Perry N, Wagaarachchi P, Robertson SA, Print CG and Hull LM: MicroRNA-regulated pathways associated with endometriosis. Mol Endocrinol 23: 265-275, 2009.

9. Pan Q, Luo X, Toloubeydokhti T and Chegini N: The expression profile of micro-RNA in endometrium and endometriosis and the influence of ovarian steroids on their expression. Mol Hum Reprod 13: 797-806, 2007.

10. Chen K and Rajewsky N: The evolution of gene regulation by transcription factors and microRNAs. Nat Rev Genet 8: 93-103, 2007.

11. Lv J, Fu Z, Shi M, Xia K, Ji C, Xu P, Lv M, Pan B, Dai L and Xie H: Systematic analysis of gene expression pattern in has-miR-760 overexpressed resistance of the MCF-7 human breast cancer cell to doxorubicin. Biomed Pharmacother 69: 162-169, 2015.

12. Huang W, Sherman BT and Lempicki RA: Systematic and integrative analysis of large gene lists using DAVID bioinformatics resources. Nat Protoc 4: 44-57, 2009.

13. Yu J, Wang Y,Zhou WH, Wang L, He YY and Li DJ: Combination of estrogen and dioxin is involved in the pathogenesis of endometriosis by promoting chemokine secretion and invasion of endometrial stromal cells. Hum Reprod 23: 1614-1626, 2008. 
14. De La Garza EM, Binkley PA, Ganapathy M, Krishnegowda NK Tekmal RR, Schenken RS and Kirma NB: Raf-1, a potential therapeutic target, mediates early steps in endometriosis lesion development by endometrial epithelial and stromal cells Endocrinology 153: 3911-3921, 2012.

15. Li MQ, Li HP, Meng YH, Wang XQ, Zhu XY, Mei J and Li DJ: Chemokine CCL2 enhances survival and invasiveness of endometrial stromal cells in an autocrine manner by activating Akt and MAPK/Erk1/2 signal pathway. Fertil Steril 97: 919-929, 2012.

16. Tapia-Pizarro A, Argandoña F, Palomino WA and Devoto L: Human chorionic gonadotropin (hCG) modulation of TIMP1 secretion by human endometrial stromal cells facilitates extravillous trophoblast invasion in vitro. Hum Reprod 28 . 2215-2227, 2013

17. Sun Q, Zhang J, Cao W, Wang X, Xu Q, Yan M, Wu X and Chen W: Dysregulated miR-363 affects head and neck cancer invasion and metastasis by targeting podoplanin. Int J Biochem Cell Biol 45: 513-520, 2013

18. Qiao J, Lee S, Paul P, Theiss L, Tiao J, Qiao L, Kong A and Chung DH: miR-335 and miR-363 regulation of neuroblastoma tumorigenesis and metastasis. Surgery 154: 226-233, 2013.

19. Vernet-Tomás MM, Pérez-Ares CT, Verdú N, FernándezFigueras MT, Molinero JL and Carreras R: The depolarized expression of the alpha- 6 integrin subunit in the endometria of women with endometriosis. J Soc Gynecol Investig 13: 292-296, 2006.

20. Giannelli G, Sgarra C, Di Naro E, Lavopa C, Angelotti U, Tartagni M, Simone O, Trerotoli P, Antonaci S and Loverro G: Endometriosis is characterized by an impaired localization of laminin-5 and alpha3beta1 integrin receptor. Int J Gynecol Cancer 17: 242-247, 2007.

21. Lin SC, Wang CC, Wu MH, Yang SH, Li YH and Tsai SJ: Hypoxia-induced microRNA-20a expression increases ERK phosphorylation and angiogenic gene expression in endometriotic stromal cells. J Clin Endocrinol Metab 97: E1515-E1523, 2012.

22. Wu MH, Wang CA, Lin CC, Chen LC, Chang WC and Tsai SJ: Distinct regulation of cyclooxygenase-2 by interleukin-1beta in normal and endometriotic stromal cells. J Clin Endocrinol Metab 90: 286-295, 2005

23. Carli C, Metz CN, Al-Abed Y, Naccache PH and Akoum A: Up-regulation of cyclooxygenase-2 expression and prostaglandin $\mathrm{E}_{2}$ production in human endometriotic cells by macrophage migration inhibitory factor: involvement of novel kinase signaling pathways. Endocrinology 150: 3128-3137, 2009

24. Tamura M, Sebastian S, Yang S, Gurates B, Fang Z and Bulun SE: Interleukin-1beta elevates cyclooxygenase-2 protein level and enzyme activity via increasing its mRNA stability in human endometrial stromal cells: an effect mediated by extracellularly regulated kinases 1 and 2. J Clin Endocrinol Metab 87: 3263-3273, 2002.

25. Chuang PC, Sun HS, Chen TM and Tsai SJ: Prostaglandin $E_{2}$ induces fibroblast growth factor 9 via EP3-dependent protein kinase Cdelta and Elk-1 signaling. Mol Cell Biol 26: 8281-8292, 2006.

26. Xu X, Zheng Q, Zhang Z, Zhang X, Liu R and Liu P: Periostin enhances migration, invasion, and adhesion of human endometrial stromal cells through integrin-linked kinase 1/Akt signaling pathway. Reprod Sci 22: 1098-1106, 2015.
27. Li J, Zhang H, Wu J, Guan H, Yuan J, Huang Z and Li M: Prognostic significance of integrin-linked kinasel overexpression in astrocytoma. Int J Cancer 126: 1436-1444, 2010.

28. Yin X, Pavone ME, Lu Z, Wei J and Kim JJ: Increased activation of the PI3K/AKT pathway compromises decidualization of stromal cells from endometriosis. J Clin Endocrinol Metab 97: E35-E43, 2012

29. Samartzis EP, Noske A, Dedes KJ, Fink D and Imesch P: ARIDIA mutations and PI3K/AKT pathway alterations in endometriosis and endometriosis-associated ovarian carcinomas. Int J Mol Sci 14: 18824-18849, 2013.

30. Franco-Murillo Y, Miranda-Rodríguez JA, Rendón-Huerta E, Montaño LF, Cornejo GV, Gómez LP, Valdez-Morales FJ, Gonzalez-Sanchez I and Cerbón M: Unremitting cell proliferation in the secretory phase of eutopic endometriosis: involvement of pAkt and pGSK3 $\beta$. Reprod Sci 22: 502-510, 2015.

31. Pan $Q$ and Chegini N: MicroRNA signature and regulatory functions in the endometrium during normal and disease states. Semin Reprod Med 26: 479-493, 2008.

32. Harada T, Taniguchi F, Izawa M, Ohama Y, Takenaka Y, Tagashira Y, Ikeda A, Watanabe A, Iwabe T and Terakawa N: Apoptosis and endometriosis. Front Biosci 12: 3140-3151, 2007.

33. Brosens JJ and Gellersen B: Death or survival - progesterone-dependent cell fate decisions in the human endometrial stroma. J Mol Endocrinol 36: 389-398, 2006.

34. Kao LC, Tulac S, Lobo S, Imani B, Yang JP, Germeyer A, Osteen K, Taylor RN, Lessey BA and Giudice LC: Global gene profiling in human endometrium during the window of implantation. Endocrinology 143: 2119-2138, 2002.

35. Jovanovic $M$ and Hengartner MO: miRNAs and apoptosis: RNAs to die for. Oncogene 25: 6176-6187, 2006.

36. Matsubara H, Takeuchi T, Nishikawa E, Yanagisawa K, Hayashita Y, Ebi H, Yamada H, Suzuki M, Nagino M, Nimura Y, et al: Apoptosis induction by antisense oligonucleotides against miR-17-5p and miR-20a in lung cancers overexpressing miR-17-92. Oncogene 26: 6099-6105, 2007.

37. Mertens-Talcott SU, Chintharlapalli S, Li X and Safe S: The oncogenic microRNA-27a targets genes that regulate specificity protein transcription factors and the G2-M checkpoint in MDA-MB-231 breast cancer cells. Cancer Res 67: 11001-11011, 2007.

38. Xu YL, Wang DB, Liu QF, Chen YH and Yang Z: Silencing of cofilin-1 gene attenuates biological behaviours of stromal cells derived from eutopic endometria of women with endometriosis. Hum Reprod 25: 2480-2488, 2010.

39. Absenger Y, Hess-Stumpp H, Kreft B, Krätzschmar J, Haendler B, Schütze N, Regidor PA and Winterhager E: Cyr61, a deregulated gene in endometriosis. Mol Hum Reprod 10: 399-407, 2004.

40. Hull ML, Escareno CR, Godsland JM, Doig JR, Johnson CM, Phillips SC, Smith SK, Tavaré S, Print CG and Charnock-Jones DS: Endometrial-peritoneal interactions during endometriotic lesion establishment. Am J Pathol 173: 700-715, 2008. 